\title{
CD8+ iT cell, a budding star for cancer immunotherapy
}

\author{
Ling Xu • Zhenyi Jin • Yangqiu Li $(\mathbb{D}$
}

Received: 8 July 2018 / Accepted: 30 July 2018 / Published online: 10 August 2018

(C) Springer Nature B.V. 2018

\begin{abstract}
Abbreviations
ACT Adoptive cellular therapy

CAR-T Chimeric antigen receptor expressing T cell

T-iPSC T cell-induced pluripotent stem cell

iHSC Induced hematopoietic stem cells

TCR T cell receptor

ETPs Early T cell lineage progenitor-like cells

DLL1/4 Notch receptor ligand Delta-like 1/4
\end{abstract}

The fate of hematopoietic cell can be modified or totally overset by genetic engineering technique. Attempts to convert B into T cells by silencing "master genes" of the B cell lineage have had limited success (Cobaleda et al.

L. Xu $\cdot$ Y. Li

Department of Hematology, First Affiliated Hospital, Jinan

University, Guangzhou 510632, China

L. Xu

e-mail: lingxu114@163.com

L. Xu • Z. Jin · Y. Li $(\bowtie)$

Key Laboratory for Regenerative Medicine of Ministry of Education, Institute of Hematology, School of Medicine, Jinan University, No.601 West of Huangpu Avenue,

Guangzhou 510632, China

e-mail: yangqiuli@hotmail.com

Z. Jin

e-mail: jinzhenyijnu@163.com

Z. Jin

Integrated Chinese and Western Medicine Postdoctoral Research Station, Jinan University, Guangzhou 510632, China
2007; Ungerback et al. 2015). Recently, a report showed that expression of hematopoietic stem cell-specific transcription factor Homeobox B5 (Hoxb5) in mouse B cell progenitors successfully reprogramed those cells into functional $\mathrm{T}$ cells in vivo. This de novo approach is potentially capable to generate $\mathrm{T}$ cells for adoptive $\mathrm{T}$ cell therapy (ACT) in future. Here, we summarized a brief commentary on the application prospect of this induced functional T cell (iT) generation strategy by this reprogramming approach.

Chimeric antigen receptor T cells (CAR-T) and T cell receptor T cells (TCR-T) are promising forms of immunotherapy against leukemia and solid tumors (Im and Pavletic 2017; Lin et al. 2017; Kasakovski et al. 2018). However, conventional CAR-T and TCR-T cell products are mainly derived from the autologous peripheral blood of patients, whose $\mathrm{T}$ cells occasionally showed functional exhaustion and senescence and reduced response to activation, which were correlated with patient's age, tumor burden, or microenvironment (Crespo et al. 2013; Vicente et al. 2016; Lin et al. 2017; Kasakovski et al. 2018). In order to overcome difficulties faced in CAR-T and TCR-T production, which were caused by the limited quantity, function 
impairment, and the terminally differentiated state of autologous $T$ cells from patients, an alternative approach has been attempted in the $T$ cell regenerative filed by generating tumor-targeted $\mathrm{T}$ cells from induced pluripotent stem cells (iPSCs), which are reprogrammed from somatic cells, such as peripheral blood cells and even T cells (Loh et al. 2010; Themeli et al. 2013; Vizcardo et al. 2013).

Published studies have shown that pro-B cells are highly plastic for reprogramming (Xie et al. 2004; Cobaleda et al. 2007), and these cells have been successfully converted into $\mathrm{T}$ cells by dedifferentiation to uncommitted blood progenitors (Cobaleda et al. 2007; Ungerback et al. 2015). Recently, Wang and colleagues reported that iT cells can be generated by the expression of hematopoietic stem cell (HSC)-specific transcription factor Hoxb5 in propre-B cells in vivo. Surprisingly, Hoxb5 directly reprogrammed $\mathrm{B}$ cells into early $\mathrm{T}$ cell lineage progenitor-like cells (ETPs) by a trans-differentiation process, since no donor-derived uncommitted hematopoietic progenitor cells were detected in the recipient mice. The induced ETPs in bone marrow can further complete mature $\mathrm{T}$ cell development in the thymus microenvironment and finally express polyclonal TCR $\beta$ chains. The mature $\mathrm{T}$ cell derivatives were capable of secreting IL-2, IL-10, IFN- $\gamma$, and TNF cytokines in response to monoclonal anti-CD3 and anti-CD28 antibody stimulation and capable of proliferating after allogenic cell stimulation in vitro. Furthermore, in vivo study identified that these iT cells rejected allogenic skin graft and formed adaptive immunological memories (Zhang et al. 2018). Despite the efficiency of lineage conversion being currently low (1\%), pro-pre-B cells expressing retro-Hoxb5 failing to generate ETPs were still able to differentiate normally into B cells, which reduces the risk of generating unknown pluripotency cells with tumor potential. It has been considered as a potential risk of iT cells by reprogramming and induction approaches (Loh et al. 2010; Vizcardo et al.

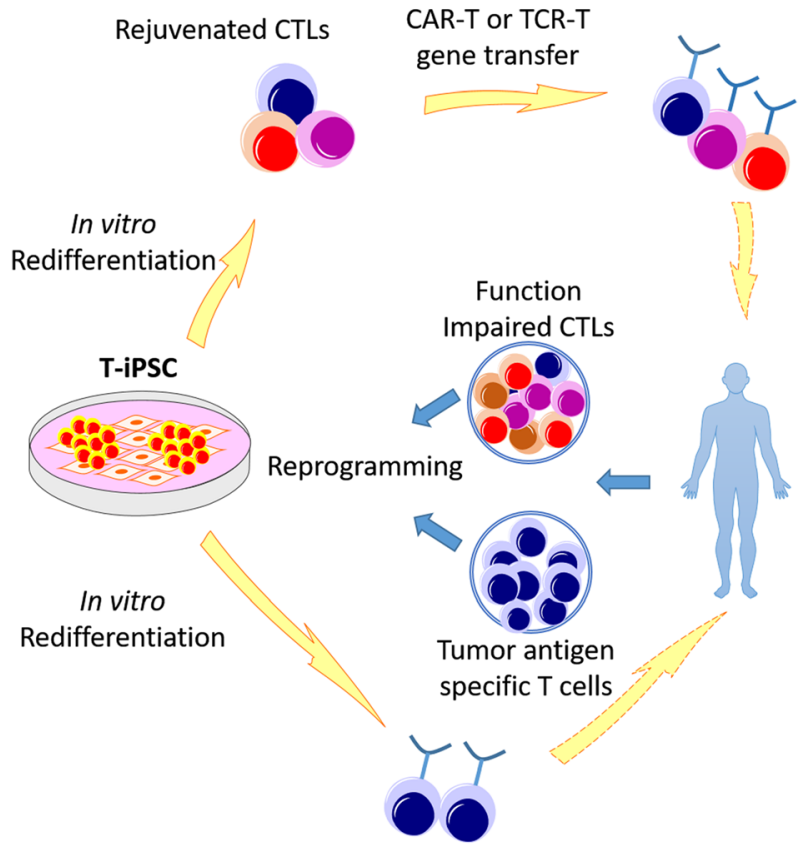

Fig. 1 Schematic diagram of promising methods to generate reprogrammed $\mathrm{T}$ cells for adoptive cellular therapy (ACT). Function impaired polyclonal CTLs isolated from tumor or leukemia patients can be reprogrammed to a pluripotent state (T-iPSC) combined with subsequent ex vivo re-differentiation and genetic modification to express antitumor $\mathrm{T}$ cell receptors or chimeric antigen receptors, these cells will be potential for ACT of tumor; CTLs carrying tumor antigen-specific TCR from patients also can be reprogrammed for ACT (left panel). In patients who lack enough $\mathrm{T}$ cells for reprogramming, rejuvenated iTs generating

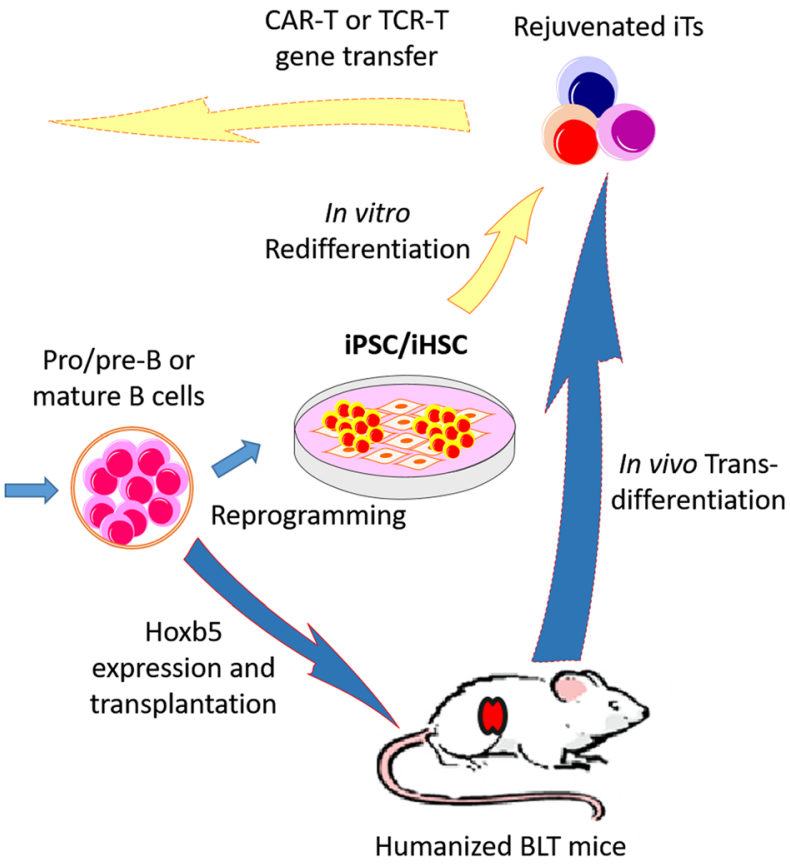

from B cell reprogrammed iPSC or iHSC can be an alternative source for ACT. Recently, retro-Hoxb5-expressing propre-B cell reprogramming creates a brand new way to generate rejuvenated iTs for ACT; however, this new method may need humanized BLT mice to provide suitable microenvironment for early $\mathrm{T}$ progenitor cells trans-differentiation and for $\mathrm{T}$ cell maturation (right panel). T-iPSC $\mathrm{T}$ cell induced pluripotent stem cell, iHSC induced hematopoietic stem cells. BLT mice, NSG mice transplanted with human thymus, fetal liver and $\mathrm{CD} 34^{+} \mathrm{HSCs}$ 
2013). In addition, the shorter lifespan of those iT cells also decreased the tumorigenesis risk compared with those less differentiated cell types. Thus, CD8+ iT cells generated by this single transcription factor reprogramming might be potentially applied to treat lymphocytopenia-related autoimmune deficiency diseases. Particularly, when combined with antitumor-specific TCR gene or CAR-T gene transfer, they could be one possible source of $\mathrm{T}$ cell generation for treating metastatic cancer and virus infection, especially for who do not have enough natural tumor- or virus-specific $\mathrm{T}$ cells for ACT.

Undoubtedly, this de novo type of CD8+ iT cells is a budding star for cancer immunotherapy. Several challenges remain to be addressed before clinical application of the reprogrammed T cells into immunotherapy. For example, when they are applied in TCR-T production, methods for endogenous TCR gene silence or modification on the transferred TCR gene should be considered to avoid the mispairing of transferred and endogenous TCR chains, which may resulting in unspecific reactivity (Cobaleda et al. 2007). In addition, reprogramming efficiency needs further improvement to meet the requirement of bulk cell numbers for ACT. For the purpose of more convenient and fast generation of T cells for clinical usage, it is necessary to indicate that HOXB5-expressing human B cells can be successfully converted into $\mathrm{T}$ cells in the humanized mice model transplanted with human thymus, fetal liver, and HSCs (BLT mice). Alternatively, an ex vivo iTcell induction system whether can be established in the presence of stromal cells expressing notch receptor ligand Delta-like 1 (Dll1) or D114. If these issues could be solved, the ACT approach using autologous ihCD8+T cells from lineage conversion will be worth an attempt. Promising methods to generate reprogrammed T cells for ACT including the new reprograming method are summarized in the Fig. 1.

\section{Conclusions}

Generating fully functional $\mathrm{T}$ cells in vivo by ectopic expression of HSC transcription factor Hoxb5 in propre-B cells holds great promise for treating patients who suffer with $\mathrm{T}$ cell immune deficiency diseases in the future. Combined with CAR and tumor antigenspecific TCR gene transfer techniques, they are also promising in cancer immunotherapy; however, a lot of challenges are still facing before arrive at the real clinical transformation.
Author contributions YQL designed the review and helped to modify the manuscript. LX drafted the manuscript and prepared the figures. ZYJ drafted the manuscript. All authors read and approved the final manuscript.

Funding This study was supported by grants from the National Natural Science Foundation of China (Nos. 91642111, 81770152), the Guangdong Provincial Basic Research Program (No. 2015B020227003), the Guangdong Provincial Applied Science and Technology Research \& Development Program (No. 2016B020237006) and the Guangzhou Science and Technology Project (Nos. 201510010211, 201807010004 and 201803040017).

\section{Compliance with ethical standards}

Competing interests The authors declare that they have no competing interests.

\section{References}

Cobaleda C, Jochum W, Busslinger M. Conversion of mature B cells into $T$ cells by dedifferentiation to uncommitted progenitors. Nature. 2007;449(7161):473-7. https://doi.org/10.1038 /nature06159.

Crespo J, Sun H, Welling TH, Tian Z, Zou W. T cell energy, exhaustion, senescence, and stemness in the tumor microenvironment. Curr Opin Immunol. 2013;25(2):214-21.

Im A, Pavletic SZ. Immunotherapy in hematologic malignancies: past, present, and future. J Hematol Oncol. 2017;10(1):94.

Kasakovski D, Xu L, Li Y. T cell senescence and CAR-T cell exhaustion in hematological malignancies. J Hematol Oncol. 2018;11(1):91.

Lin C, Chen S, Li Y. T cell modulation in immunotherapy for hematological malignancies. Cell Biol Toxicol. 2017;33(4):323-7.

Loh YH, Hartung O, Li H, Guo C, Sahalie JM, Manos PD, et al. Reprogramming of T cells from human peripheral blood. Cell Stem Cell. 2010;7(1):15-9.

Themeli M, Kloss CC, Ciriello G, Fedorov VD, Perna F, Gonen $\mathrm{M}$, et al. Generation of tumor-targeted human T lymphocytes from induced pluripotent stem cells for cancer therapy. Nat Biotechnol. 2013;31(10):928-33.

Ungerback J, Ahsberg J, Strid T, Somasundaram R, Sigvardsson M. Combined heterozygous loss of Ebfl and Pax 5 allows for T-lineage conversion of B cell progenitors. J Exp Med. 2015;212(7):1109-23.

Vicente R, Mausset-Bonnefont AL, Jorgensen C, Louis-Plence P, Brondello JM. Cellular senescence impact on immune cell fate and function. Aging Cell. 2016;15(3):400-6.

Vizcardo R, Masuda K, Yamada D, Ikawa T, Shimizu K, Fujii S, et al. Regeneration of human tumor antigen-specific $\mathrm{T}$ cells from iPSCs derived from mature CD8(+) T cells. Cell Stem Cell. 2013;12(1):31-6.

Xie H, Ye M, Feng R, Graf T. Stepwise reprogramming of B cells into macrophages. Cell. 2004;117(5):663-76.

Zhang M, Dong Y, Hu F, Yang D, Zhao Q, Lv C, et al. Transcription factor Hoxb5 reprograms B cells into functional T lymphocytes. Nat Immunol. 2018;19(3):279-90. 